\title{
The flapless air vehicle integrated industrial research (FLAVIIR) programme in aeronautical engineering
}

\author{
P John \\ FLAVIIR Programme, School of Engineering, Cranfield University, Cranfield, Bedfordshire MK4 30AL, UK. \\ email: P.John@cranfield.ac.uk
}

The manuscript was received on 6 April 2009 and was accepted after revision for publication on 18 August 2009.

DOI: 10.1243/09544100JAERO580

\begin{abstract}
This article describes an exciting research programme, FLAVIIR (flapless air vehicle integrated industrial research), in Aeronautical Engineering that is developing and demonstrating novel technologies and also forging a new approach to academic research, with emphasis on engagement with, and relevance to, industry. Traditional academic research is usually focused on fundamental properties of technologies, proof of principles, and solution concept generation. This leaves industry to bridge the gap between this foundation work undertaken by the academic research and the introduction of technologies into practical products suitable for use in the real world. The FLAVIIR programme is taking academic research further than usual so that it relates more closely to technology exploitation in industry and so makes a significant contribution to the practical realization of novel technologies, so increasing the benefits delivered to industry from investment in academic research. In order to achieve this, the FLAVIIR programme includes, in addition to research into the fundamental technologies themselves, an emphasis on technology integration into an air vehicle that is then used to demonstrate the technologies in a representative manner. The FLAVIIR programme is enabled and funded by a strategic partnership formed between BAESystems and the Engineering and Physical Sciences Research Council (EPSRC) in Aeronautical Engineering.
\end{abstract}

Keywords: systems engineering, uninhabited air vehicle, technology readiness levels, university research, industrial collaboration

\section{INTRODUCTION}

An organization such as BAESystems, with its complex, high technology, high-performance system products, relies on high quality research to drive the innovation that underpins its business. The company has worked closely with the academic sector for many years to provide technology innovations which it has then sought to take forward into its practical, integrated system products. The FLAVIIR (flapless air vehicle integrated industrial research) research programme represents an innovative approach to industrial-academic research collaboration [1]. There are several aspects to the innovative approach. First, a strategic agreement was established between BAESystems, the major international aerospace company, and the UK's Engineering and Physical Sciences Research Council (EPSRC) in which the two organizations agreed to jointly fund a major research programme in Aeronautical
Engineering. This partnership is intended to stimulate academic research that is better suited to the needs of industry. In particular, the objective of the programme is to develop a research capability in UK academia that is more industrially focused and relevant than has been the case traditionally. This strategic partnership funds research through a collaborative engagement with several chosen academic network partners across the UK, led by Cranfield University as the chosen 'Strategic Capability Partner'. These relationships are illustrated in Fig. 1.

A total of 14 research groups at ten UK universities are involved in the programme, which has a total value of $£ 6.5 \mathrm{M}$. The 5-year programme started in 2004 and runs until December 2009.

This innovative joint approach to funding research in academia drives increased collaboration between the academic institutions and an emphasis on achieving an integrated outcome of practical relevance for 


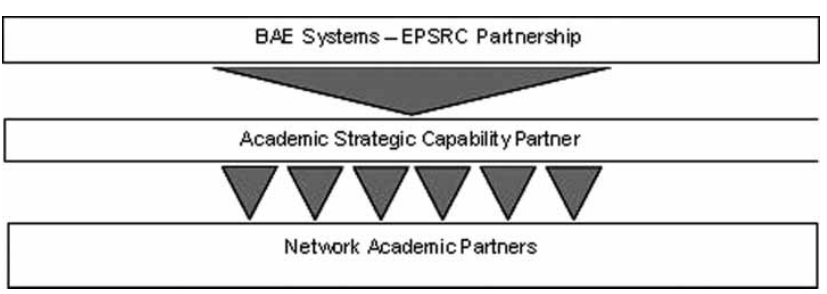

Fig. 1 Schematic representation of the BAESystems EPSRC - university partnerships

industrial exploitation. Hence the innovation of the research programme can be summarized by the key words of 'Collaboration', representative 'Integration', and 'Exploitation'. The Strategic Capability Partnership in Aeronautical Engineering, with the associated FLAVIIR research programme, was the first such relationship to be launched by BAESystems. Similar strategic relationships and research programmes have since been launched in other disciplines. These include Systems Engineering, with the NECTISE research programme (Network Enabled Capability Through Innovative Systems Engineering) [2] and Information Systems, with the ALADDIN research programme (Autonomous Learning Agents for Decentralised Data and Information Networks) [3].

\section{TECHNOLOGY READINESS LEVELS}

The increased emphasis on industrial relevance and exploitation of novel technologies can be expressed in terms of 'Technology Relevance Levels' (TRLs), the aim being to take academic research to higher TRLs than is usually the case. TRLs are usually described through a scale of nine levels as follows.

1. TRL9: Actual technology/system qualified through successful mission operations.

2. TRL8: Actual technology/system completed and qualified through test and demonstration.

3. TRL7: Technology/system prototype demonstration in an operational environment.

4. TRL6: Technology/system/subsystem model or prototype demonstration in a relevant environment.

5. TRL5: Technology component and/or basic technology subsystem validation in relevant environment.

6. TRL4: Technology component and/or basic technology subsystem validation in laboratory environment.

7. TRL3: Analytical and experimental critical function and/or characteristic proof-of-concept.

8. TRL2: Technology concept and/or application formulated.

9. TRL1: Basic principles observed and reported.
Broadly speaking, academic research is often focused on fundamentals of technologies and proof of concept, as indicated by TRLs 1 to 3 . On the other hand, industry, in developing system products that are practicable, proven and certified for use in the real world, must operate through TRLs 7 and 8 up to TRL 9. Usually it falls to industry to bridge this gap through TRLs 4 to 6 , which is sometimes dramatically called the TRL 'Valley of Death' in view of the many technologies that fail to reach their potential through successful exploitation in industrial products and processes. In FLAVIIR, the aim is for academia to move into undertaking these higher levels of research and so provide a capability to industry that is of more relevance to exploitation in their products. One important feature of the TRLs defined above is that higher TRLs relate to technologies being used in increasingly representative contexts and environments. This includes increasing the level of integration of technologies with others associated with their overall real world operation in products. Hence this aim of FLAVIIR requires that research into individual technologies, conducted by individual university research teams, is extended to multidisciplinary teamworking on integrated technology concepts, designs, and demonstrators. Only through such collaboration can the practical implications, design trade-offs, and overall system behaviour associated with higher TRLs be investigated and demonstrated. This means that the universities are engaged in systems integration as well as in the technology research and so develop skills and knowledge that are usually restricted to industry.

\section{GRAND CHALLENGES AND RESEARCH THEMES}

In order to stimulate the above research approach, two 'Grand Challenges' were set for the FLAVIIR programme:

(a) 'a maintenance-free UAV without conventional control surfaces and no cost or performance penalties';

(b) 'significant research impact through effective academic/industry management and exploitation of large-scale, integrated academic research'.

The first of these sets out the technical challenge to be tackled within the field of Aeronautical Engineering: to research the achievement of an uninhabited air vehicle with novel control surface technologies and with performance and cost comparable or better than conventional technologies. This results from a drive to reduce the acquisition and operation costs of UAVs while retaining capability. The advantages of unmanned systems must be demonstrated in terms of performance ('effectiveness' is often used as a measure for military aircraft) but it should also be recognized 
that this effectiveness is quickly eroded if the costs of acquisition or operation are too high. As an example, it has been reported in the past that at one stage the rate of loss of the Predator UAV was 32 per 100000 flying hours and this was an order of magnitude higher than the more capable F-16 [4]. Such expensive losses diminish the cost effectiveness of the unmanned system and make it less attractive to the end customer.

The second sets out the aspiration of establishing a new research relationship between industry and academia and across academia focused on higher TRLs and exploitation of the research.

Early in the FLAVIIR programme, key areas of research associated with the first of the Grand Challenges mentioned above were identified and selected. These areas encompassed technologies that would support the achievement of low cost, flapless unmanned aerial vehicles (UAVs) and were expressed as the following Research Themes.

1. Aerodynamics, with research groups at Cranfield University, Imperial College of Science, Technology \& Medicine, and the University of Manchester.

2. Control systems, with research groups at the University of Leicester, Imperial College of Science, Technology \& Medicine, and Cranfield University.

3. Electromagnetics, with research groups at the University of Nottingham, the University of Wales (Swansea), and the University of York.

4. Manufacturing, with research groups at Warwick University, the University of Liverpool, and Cranfield University.

5. Materials and structures, with a research group at Imperial College of Science, Technology \& Medicine.

6. Numerical simulation, with a research group at Southampton University.

In addition, to meet the second Grand Challenge, the design, manufacture, integration, and flight of a demonstrator uninhabited air vehicle will provide the research teams with an integrated platform on which to evaluate the performance of their technology in a realistic flight environment. This is encapsulated in a research theme of:

1. Integration and demonstration, with multi-university collaboration led by a research group at Cranfield University.

The inclusion of this technology demonstrator in the programme is an important feature. A report from the Aerospace Innovation and Growth Team (AeIGT) on the future of the UK aerospace industry [5] highlighted the historical success of demonstrator programmes within the aerospace sector and recommended their adoption to improve the UK's competitive situation for the future.

\section{RESEARCH SUMMARY}

In this Special Edition of this journal several papers are presented that describe some of the research that has been conducted on the FLAVIIR programme in some of the Research Themes mentioned above. The following sections provide an overview of the research conducted on the FLAVIIR programme by summarizing the principal focus of the research conducted in each of the themes.

\subsection{Aerodynamics}

The main focus of the aerodynamics research is on novel means of flow control for improved manoeuvrability, design robustness, and simplicity. Technologies are being investigated that will replace conventional control flaps with fluidic devices that achieve aerodynamic control without the need for moving surfaces. Manchester and Cranfield are investigating roll control using wing-mounted fluidic devices that use pressurized air from slots that extend along the trailing edge of a UAV wing. The basic principle is that blown air entrains the upper surface flow over the wing, thus giving rise to an increase in lift. The trailing edge has to be modified to become rounded, but in general the thickness of a typical trailing edge does not have to be altered. Manchester are also investigating pitch control using vectoring of the principal thrust from the main gas turbine engine of a UAV. The thrustvector control device is mounted around the jet from a gas turbine engine with upper and lower slots that produce secondary Coanda pressurized jets that are controlled to cause deflection of the primary engine jet. The principle is that deflection of the main jet can be achieved by switching on a higher pressure secondary wall jet that adheres to the geometry and deflects the main jet with it.

\subsection{Control systems}

The Control System research is providing technologies that will support increased levels of autonomy, provide the ability to reconfigure to react to a changing environment, and aid integration into a distributed control network. Advances investigated in this area include coordinated control, integrated control (multi-platforms) and condition monitoring; nonlinear and adaptive control, distributed aerodynamic control.

Leicester have focused on coordinated, integrated, and distributed control. Imperial College have studied adaptive and non-linear control strategies for systems. This will be focused on devising new generic adaptive and non-linear control algorithms suited to aerospace applications and the provision of robust but nonconservative implementations of these algorithms. 
The end goal is improved performance and reliability for the control software.

\subsection{Electromagnetics}

The thrust of the electromagnetic theme is to be able to predict and find novel ways of reducing susceptibility of composite material structures to radio frequency (RF) threats and lightning strike. A single predictive code has been produced collaboratively by three Universities (Swansea, Nottingham, and York). Swansea have applied novel numerical analysis techniques utilizing fast hybrid mesh methods. Nottingham have researched ways to model small-scale electrical devices within a large-scale environment in an efficient manner. The aim is to be able to efficiently and accurately design resilient composite material structures with small gaps, slots, cables, and wires. Such a tool will allow more detail and novel ideas to be explored at the concept stage. The research at York has concentrated on material models: how to model carbon fibre structures, honeycombs, etc. and on determining the effects of manufacturing irregularities on electromagnetic fields during pulsed RF threat and lightning strike.

\subsection{Manufacturing}

The focus of the manufacturing research is on reducing cost, not only in production but also in reducing maintenance and increasing reliability. Warwick have studied areas such as low cost reconfigurable tooling; Cranfield have studied novel manufacturing processes for composite materials, such as dry fabric laminating, trans-laminar reinforcement, single sided stitching and tufting. Liverpool have researched Direct-write technologies and methods, with functional elements being achieved on and within composite structures by laying down conductive tracks directly onto the material using printing technologies, special inks, and laser curing.

In addition, the Cranfield Manufacturing team has manufactured the composite material airframe of the DEMON aircraft, which has been developed to be the FLAVIIR demonstration UAV.

\subsection{Materials and structures}

The materials research has focused on developing predictive methods to model delamination in composite materials. An accurate numerical method has the potential to provide substantial cost savings by a simple reduction in the number of component tests that currently have to be completed. Imperial College have demonstrated the potential of a novel 'interface element' method that allows easy and accurate modelling of such failure mechanisms.

\subsection{Numerical simulation}

The numerical simulation work within FLAVIIR is concerned with numerical design and optimization. Southampton have formulated a concept design framework that enables modelling of complexity and cost within a concept design. These geometry-based designs (using CAD descriptions) are coupled to various optimization strategies to allow 'what-if' studies to be undertaken. Cost modelling has been investigated not only to obtain the cost of parts but also in obtaining the cost of integration based on part complexity, function, and mission importance.

\subsection{Integration and demonstration}

Integration and demonstration are crucial elements within the FLAVIIR research in order to achieve the aim of increasing the 'TRL' achieved by academic research. Technologies and research from across the programme are brought together in a collaboration to design, build, integrate, and fly a representative uninhabited air vehicle, called 'DEMON'. This demands an integrated multidisciplinary design approach, with complex trade-offs and risks to be dealt with in order to incorporate the novel technologies from the programme into a practical, integrated aircraft that will be flown to demonstrate the technologies in real world action. Cranfield are leading the Integration and Demonstration research and this will provide invaluable information on the performance of the novel technologies as well as exercising a multidisciplinary teamwork approach centred on system integration and proving.

\section{EXPERIENCES AND LESSONS LEARNT}

The FLAVIIR programme is aiming to achieve innovation in a number of ways. The novel research encompasses a very wide range, from technologies for flight control (hardware and software) to manufacturing processes, to methods to support improvements in the design process, such as optimization and predictive techniques. Further, the programme's focus on increasing Technology Readiness Levels, and the corresponding focus on the Integration and Demonstration of a representative Uninhabited Air Vehicle demand that the university groups work together very closely in a multidisciplinary team dealing with complex design trade-offs and the manufacturing, assembly, proving, and certification of the vehicle. In addition, the focus on industrial relevance and exploitation requires a very close collaboration between the academics and industry and also requires an appropriate focus within industry itself. Several key lessons have been learned through the experiences of 
dealing with these issues on the FLAVIIR programme and these are described below.

\subsection{University approaches and capabilities}

One of the primary aims of FLAVIIR is to develop industrially relevant multidisciplinary skills in academia. The achievement of a real uninhabited air vehicle requires many technologies to be integrated in a practical design, with the university researchers involved needing to work together in a collaborative, multidisciplinary team. This requires skills in understanding the wider context and application into which their individual technologies are being implemented and a focus on practical issues associated with their implementation. These practical aspects include nonfunctional properties of the technologies, such as reliability, weight, and environmental characteristics (e.g. thermal properties and electromagnetic compatibilities). The researchers also need to consider the failure modes and limitations of their technologies so that the effectiveness, balance, and robustness of the overall design can be assessed throughout the design process. The integration and demonstration activities on FLAVIIR are led by a team in the Aerospace Engineering department at Cranfield University and the programme has demonstrated the importance of the team having capabilities in multidisciplinary team leadership, overall system evaluation, design, assembly, integration, and test. The decision to develop a representative flying technology demonstration aircraft as the culmination of the FLAVIIR programme has demanded that academics develop such capabilities in order to deal with the critical integration issues that arise at higher TRLs. Issues that have been crucial include the common problem of weight growth in the UAV design and control of its centre of gravity ( $\mathrm{C}$ of $\mathrm{G}$ ) position. These parameters have been recognized throughout the design process as crucial issues, linked directly to flight feasibility and performance and demanding close attention to the interdependencies between aspects such as aerodynamic performance and stability, structural integrity and design adaptability. The latter aspect of design adaptability is important in the FLAVIR DEMON technology demonstration aircraft because in order to manage risk and to provide a rigorous evaluation of novel technologies, the aircraft will be flown with new technologies introduced progressively. Hence the aircraft will first fly with conventional control mechanisms and novel mechanisms will be introduced once the baseline characteristics of the aircraft and conventional technologies are established. This progressive approach requires a modular design in order to change the technology configuration and make appropriate adjustments to the flight control system and the mass distribution within the aircraft, using flexible ballast in order to manage the change in $\mathrm{C}$ of $\mathrm{G}$ position.
Weight growth and C of G management proved to be significant challenges throughout the design process and demanded close collaboration between the technology researchers, aerodynamicists and designers of the flight control system, the aircraft structure and the overall system. Examples of other practical issues that had to be tackled include failure mode analysis of the integrated UAV design, design of the aircraft operating procedures for the demonstration flights, a safety case analysis, and negotiation with regulatory authorities to achieve appropriate flight clearance. Approval of the appropriate authorities to fly the DEMON aircraft is of course an essential consideration and raises potential challenges given the objective of making the aircraft representative of real-world issues. The DEMON design team at Cranfield developed their capabilities in this area and liaised directly with the Civil Aviation Authority to discuss flight clearance. However, since the objective is to fly the aircraft as a technology demonstrator, not as an operational vehicle in itself, it was decided to simplify the clearance issues by flying only on an approved range with segregated airspace and ground area and with the flight profile contained within an agreed safety footprint within the range boundary, so interaction with public areas is avoided. This approach reduces the need for full compliance with formal certification standards and enables a case to be made based on evaluations of the aircraft integrity, its performance and mass characteristics, the safety features, such as a cut-down system, and its operating procedures at the range. The Certification Specification for Very Light Aircraft (CS-VLA) has been used as guidance on the structural design and integrity and the CAA's 'Unmanned Aircraft System Operations in UK Airspace' (CAP 722) and 'Policy for Light UAVs' have been used as guidance on flight operations and procedures for the DEMON aircraft.

Achieving the necessary approach across universities has required special efforts to overcome challenges and barriers. Traditional academic research tends to be focused within groups, with independent ownership of research outcomes. This is reinforced by conventional academic measures, such as publications on individual research. Academia is not structured to easily accommodate and encourage multidisciplinary research, particularly across university organizational boundaries. In the early stages of the programme, once the research themes and priority technologies and processes had been identified and agreed, the inevitable focus within each research group was on establishing the fundamental research progress in their area of expertise and this represented a familiar mode of operation for researchers. At this stage, cross-programme awareness and communication was achieved primarily through the formation of a Technical Committee, chaired by the Technical Director and with membership comprising 
the research leaders from each university group and corresponding lead representative from BAESystems for each technical theme. This committee met several times per year. In addition, an annual programme conference was held to enable researchers to present their research to each other and to invited industrialists. These mechanisms were always recognized as inadequate to achieve the more integrated approach necessary to achieve the overall FLAVIIR objectives and so, as the programme progressed and the individual research technologies were increasingly needing to be focused on the 'integration' and 'higher TRL' objective, a different approach was introduced. Stimulated by the challenge of designing an integrated UAV to demonstrate the technologies working successfully together, weekly integration meetings were initiated at approximately the mid-point of the programme. These meetings involved the researchers from the university groups associated with the technologies that were to be integrated into the 'DEMON' UAV, and quickly exposed the practical issues, interdependencies, and design trade-offs that lie at the heart of increasing the TRL. The meetings were held face-toface for a period of approximately 12 months and then, as the understanding of the integration issues matured, the meetings were held over the internet using Webex technology. FLAVIIR has succeeded in building an integrated team approach around the tangible integration, demonstration, and TRL challenges prompted by the development and flight of the DEMON aircraft. Without such a stimulus then it would have been much more difficult to encourage the overall system, higher TRL capabilities, and multidisciplinary teamworking and it is very unlikely that the FLAVIIR objectives would have been achieved. As it stands, everyone involved in the DEMON UAV development has gained a great deal of experience in such capabilities.

\subsubsection{Challenges faced in relation to university capabilities}

Traditional academic research and the capabilities associated with it do not focus on the practical issues that arise at higher TRLs. Academia is not organized along multidisciplinary lines.

\subsubsection{Key lessons learnt in relation to university capabilities}

The capabilities to tackle these issues can be developed within academia but a shared tangible outcome that stimulates the representative, integrated issues is invaluable, if not essential in order to stimulate the necessary multidisciplinary teamworking and practical outcome-driven focus. The DEMON UAV development not only provided the platform for demonstrating higher TRLs but also the integrated outcome focus for developing the academic capabilities. As in any development programme, frequent integration meetings and shared decision making are essential and the academics involved in DEMON gained greatly by adopting such approaches.

\subsection{Achieving an enduring university capability}

The long-term aim of the strategic partnership between BAESystem, the EPSRC, and the universities involved in FLAVIIR is to develop an enduring academic capability that is capable of engaging with industry at higher levels of TRL. One important lesson learnt from FLAVIIR, which seems obvious in hindsight, is that the expertise and experience that is gained should be primarily vested in the permanent academic staff and not predominantly in the postdoctoral research officers and doctoral students on the programme, who tend to move on more often in these early stages of their career, thus compromising the sustainable capability. It is the academics who will form the principal enduring resource in a given university, to retain and further develop the expertise and so be able to continue to deliver and improve the intended industrially focused capability. However, the FLAVIIR programme has faced a significant difficulty in ensuring that a strong multidisciplinary, integrated expertise is developed significantly in academic staff rather than predominantly in postdoctoral research officers. The challenge arises because the FLAVIIR programme started in 2004, before the current Full Economic Cost (FEC) rules were implemented by the EPSRC. Therefore, the FLAVIIR programme is funded through an EPSRC 'pre-FEC' grant, under whose financial terms academic staff costs are not allowed to be charged. This causes a significant difficulty because it presumes that the research can be primarily conducted by postdoctoral research officers, with occasional supervision from academics. This is not suitable for meeting the FLAVIIR integration and demonstration challenge to achieve higher TRLs and develop a sustainable academic capability. Consequently, a significant commitment has been required to ensure that academic staff have a significant involvement in the day-to-day research approach and multidisciplinary capability development. A request to the EPSRC to relax this aspect of the pre-FEC rules was declined, demanding a considerable financial investment in particular by Cranfield University as 'system integrator' for DEMON. In addition to the experience gained by academics, that gained by the research officers and $\mathrm{PhD}$ students on FLAVIIR is also of course very valuable and several have been recruited into BAESystems from FLAVIIR, thus achieving another benefit from the programme in terms of the development of capable recruits into industry. 


\subsubsection{Challenges faced in relation to an enduring academic capability}

The EPSRC grant for FLAVIIR was let under 'pre-FEC' rules that do not allow academic staff costs to be charged. However, the difficulty and novelty of the FLAVIIR integration challenge, and the objective to achieve a new, sustainable academic capability has demanded significant involvement by academics, for the reasons described above.

\subsubsection{Key lessons learnt in relation to an enduring academic capability}

The EPSRC pre-FEC rules were not really the ideal to achieve the FLAVIIR objectives because of the restrictions on the recovery of academic staff costs. Full academic involvement is essential and would be allowed under the EPSRC's current FEC rules. A significant investment by the DEMON UAV system integrator, Cranfield University, has been required to address this problem.

\subsection{Collaboration between industry and universities}

An important objective of the FLAVIIR programme has been to achieve academic research that is relevant to the needs of industry and, in the first instance, to BAESystems as the principal funding provider. (Note, however, that the route for this industrial funding is via the EPSRC and so research council grant Terms and Conditions apply, as in the 'pre-FEC' discussion in the previous section.) As explained above, this is seen in the emphasis on increased TRLs, representative technology demonstration and integration. In order to achieve this, it is clear that academic researchers and industrialists need to work closely together so that, on the one hand, the needs of industry are understood by academia and, on the other, that industry understands the outcomes of the research, both potential and emerging, and consider how best to exploit it within their activities and products. Consequently, as the FLAVIIR programme has progressed, there has been increasingly close collaboration between industry and the various academic groups. This collaboration has taken several forms.

\subsubsection{Programme collaboration}

At an overall FLAVIIR programme level, a Project manager from BAESystems is assigned to the programme part-time to work with the academic Technical Director and this has proved to be an essential link between academia and industry, particularly as the research progressed and the emphasis on exploitation grew, as described below.
In addition, there has been industrial oversight of the FLAVIIR programme by a Strategic Capability Partnership Steering Group, which is chaired by a senior executive of BAESystems and which covers all aspects of the strategic partnership in Aeronautical Engineering. Wider guidance for the programme is provided by an Independent Steering Group (ISG), chaired by a senior government or industrial executive and with members with vast experience and excellent reputation. The EPSRC is represented on the ISG. The purpose of the ISG is to provide a strategic level of guidance for the project on technical issues, and to provide the normal checks and balances for publicly funded research. The Head of University Partnerships for BAESystems sits on both of these steering groups.

\subsubsection{Research collaboration}

Throughout the programme researchers in the various specialist groups across the partner universities have worked in close collaboration with nominated technical experts from corresponding areas within BAESystems, which are organized into 'Technical Focus Groups'. This has enabled industrial advice and guidance to add value to the research content and direction and has ensured that the company is well informed of the emerging research and potential benefits. In this way the academic research on FLAVIIR can be harmonized with relevant research being conducted within the company.

\subsubsection{Integration and assembly collaboration}

Collaboration has been particularly strong in the development and assembly of the DEMON UAV itself. A 'Demon Delivery Manager' from BAESystems was assigned to the project for a period to work closely with the Integration team at Cranfield University on the integrated design and procurement for DEMON and to act as the interface with supporting company capabilities such as manufacturing. For example, some of the assembly of the aircraft itself has been conducted at the BAESystems Apprentice School. This has been of mutual benefit, providing the company's apprentices with an exciting, real aircraft project on which to develop and practice their skills while providing a cost-effective solution to the FLAVIIR need to build the DEMON aircraft.

\subsubsection{Demonstration collaboration}

The culmination of much of the FLAVIIR research is in the demonstration flights of the DEMON UAV. This representative UAV requires appropriate confidence in the design integrity, flight characteristics, and flight operations to enable the demonstration 
flights to be undertaken and to achieve the necessary level of formal flight clearance. The experience of BAESystems in real projects and, in particular, in previous UAV projects has been invaluable in supporting these issues. For example, formal Design Reviews and Flight Readiness Reviews, chaired by experienced company engineers, have provided confidence in the design; flight experts from BAESystems with experience of previous UAV programmes have collaborated on the assessment of flight characteristics, and advised on the conduct of, and clearance for, demonstration flights. Also, BAESystems are providing the experienced pilot who will control the aircraft during its non-autonomous flights.

\subsubsection{Exploitation collaboration}

As the FLAVIIR research has progressed and matured, the focus on Exploitation has increased and engagement between researchers and projects within BAESystems has been identified, facilitated, and managed. This has been enabled by the industrial focus on exploitation mentioned in the next section. In addition, since many of the details associated with exploitation are commercially sensitive and may not be made completely visible to all the academic researchers, the Project Manager mentioned above (see Programme Collaboration) has undertaken a role to work with the Exploitation manager (see below) across industry and academia to identify and build appropriate links and drive through research priorities.

\subsubsection{Challenges faced in relation to industry-} university collaboration: One of the principal objectives set out for FLAVIIR was to achieve significant research impact through effective academic/industry management and exploitation of large-scale, integrated academic research. In short, this means forging a new relationship between industry and academia in research, its relevance and exploitation. This has proved challenging because the previous relationships between industry and academia have been established around more traditional and focused research topics within technical disciplines and have had research outcomes at relatively low TRLs, with the effort to take the basic research and develop it into practical products resting with industry. There were no pre-existing routes for collaboration and appropriate engagement and so relationships and mechanisms had to be created to achieve the objective. In many ways this represents a challenge in cultural change for both industry and academia.

5.3.5.2 Key lessons learnt in relation to industryuniversity collaboration: The FLAVIIR experience has shown that it is absolutely crucial to develop close relationships between industry and academia if the nature of academic research is to be changed. The involvement of appropriate industrial people, with technical expertise, knowledge of industrial priorities, and the ability to influence internal industry attitudes towards academic research, has been crucial and FLAVIIR had to identify and implement means of achieving this. Change such as that sought by FLAVIIR cannot be achieved by an administrative or financial relationship alone; close technical and programmatic engagement is essential and, on the industrial side, this has required a strong, ongoing commitment by BAESystems. Within academia this has required close engagement by the academics in each research area with industrial technical experts and cooperation by the Technical Director and the academic research leads in shaping the research plans and emphasis in response to the priorities set by industrial relevance and needs. Flexibility and cooperation has been essential from all parties. This showed itself most vividly in the downselection of technologies that was undertaken, reducing the wide range of initial research areas that had been investigated into priority areas for investigation, notably in the DEMON technology demonstrator activities. This prioritization was driven by industrial exploitation opportunities as described below and led to several of the initial research areas not receiving FLAVIIR funding in the later stages of the programme. In all cases the cooperation and understanding of the academics was essential in maintaining good relationships. The FLAVIIR approach to industrial exploitation is such an important part of the programme that it is described further below.

\subsection{Industrial focus on exploitation of research}

The aim of FLAVIIR to engage with industry at higher TRLs is driven by an objective of taking academic research closer to industrial exploitation. In order for industry to be able to exploit the technologies arising from the research into real products, or to incorporate improvements in processes, methods, and tools arising from the research into their practices, effort is required on the part of industry to explicitly link the research outcomes to their business needs and opportunities. Often this is not done very explicitly within industry and it can even be a problem in the exploitation of in-house research, let alone external research from academia. For the FLAVIIR programme, BAESystems has fully recognized the importance of a clear focus within the company on business need and corresponding research exploitation. The company has implemented a rigorous approach of Research and Technology Roadmapping that links business need, expressed with specific projects and products as targets, to research progress and outcomes, expressed as plans for the progressive increasing of TRLs, and to programmes such as FLAVIIR that are delivering them. Such an 
approach provides an explicit basis for expressing the exploitation paths for FLAVIIR technologies, processes, and methods. In addition to using these Roadmaps to derive outcome priorities for FLAVIIR linked to clearly defined exploitation paths and targets, the company assigned a highly experienced, senior engineer as an Exploitation manager to own the identification and management of these exploitation paths. Through these initiatives, the FLAVIIR research has already linked into several real projects within BAESystems, with agreed plans for the incorporation of the research outputs into wider company projects.

\subsubsection{Challenges faced in relation to industrial exploitation of university research}

Several challenges were apparent in achieving the practical exploitation of FLAVIIR research. First, it is clearly crucial that industry itself expresses its exploitation opportunities and priorities. However, these are often linked to commercially sensitive strategies, projects, and plans and so are not easily shared widely with academia. Second, academics are primarily judged by achieving academic publications in respected journals and this is important for their career progression; a focus on industrial exploitation is not always a direct match with such criteria and so academics must be prepared to be focused on industrial exploitation and a way found to reconcile any conflict with open publication.

\subsubsection{Key lessons learnt in relation to industrial exploitation of university research}

The FLAVIIR experience has shown that making academic research more relevant to, and exploitable by, industry places obligations on both parties. Industry must develop a clear internal approach to identifying their research needs, the associated TRL development path and timescale and the target business opportunity, as well as being able to engage with academics on these issues and incorporate the academic research into their organizational approach. Academics must be able to publish their research outcomes in respected journals; in order to achieve this on FLAVIIR a review process was established whereby all universities involved signed up to appropriate non-disclosure agreements and papers were assessed by BAESystems prior to submission to ensure that no commercially sensitive content was included. However, the company did not impose unnecessary or unreasonable restrictions on academic publications during this process. This process was therefore successful in allowing academics to publish while enabling the company to be confident in sharing the level of information necessary to achieve exploitation.

\section{FINAL REMARKS}

The FLAVIIR programme is an exciting research programme that encompasses a wide range of technologies, processes, and methods. However, its objectives are much more radical than a conventional academic research programme. It is taking academic research to higher Technology Readiness Levels and closer to industrial exploitation than is traditionally the case. This has been a strong focus across the FLAVIIR programme, with academics and industrialists working together to bridge the so-called 'TRL Valley of Death'. This demands new capabilities within academia to work as multidisciplinary teams and to develop, manufacture, assemble, integrate, test, and fly a representative uninhabited air vehicle. It also demands new, close relationships between the academic researchers and industry, to collaborate on the research itself and on its business exploitation. Finally, it requires new capabilities within industry itself, with an explicit focus being required on linking research outputs to business needs and exploitation opportunities and also on the means by which the industrial projects and capabilities would accommodate the innovations arising from the research.

\section{ACKNOWLEDGEMENT}

The author acknowledges the technical and financial support for the project from BAE SYSTEMS and the EPSRC (Grant Number GR/S71552/01).

(C) Author 2010

\section{REFERENCES}

1 McDougall, N. Early experiences in collaborative industry/aeronautic research in aeronautical engineering'. AEROGRAM Magazine (Cranfield University), 2005, 11 (5), $17-19$.

2 Henshaw, M. and Gunton, D. NECTISE and the strategic partnership in systems engineering. In Proceedings of the Systems Engineering for Future Capability Conference, Loughborough University, UK, 12-13 February 2007.

3 Autonomous Learning Agents for Decentralised Data and Information Networks (www.aladdinproject.org).

4 Sweetman, B. UCAVs grow fat on requirements. Jane's International Defence Review, April 2003.

5 The Aerospace Innovation and Growth Team. An independent report on the future of the UK aerospace industry volume B overview'. published by the DTI, London, 2003. 